\section{An outpatient geriatric evaluation and management programme was more effective than usual care in preventing functional decline in high risk older adults} Boult C, Boult LB, Morishita L, et al. A randomized clinical trial of outpatient geriatric evaluation and management. J Am
Geriatr Soc 2001 Apr;49:351-9.

\section{QUESTIONS: Is an outpatient geriatric evaluation and management (GEM) programme more effective than usual care in preventing functional decline in high risk older adults? Do use and cost of healthcare services differ between groups?}

\section{Design} assessors), controlled trial with 18 months of follow up.

\section{Setting}

Ramsey County and adjacent zip codes, Minnesota, USA.

\section{Patients \\ 568 patients (mean age 79 y, $56 \%$ men, $96 \%$ white) who were $\geqslant 70$ years of age, community dwelling, fee for service Medicare beneficiaries, and at high risk for hospital admission and functional decline. Exclusion criteria included residence in a nursing home, illness requiring frequent physician visits, and communication barriers. Study participants $(96 \%)$ or their proxies $(4 \%)$ completed $97 \%$ of follow up interviews.}

\section{Intervention}

294 patients were allocated to the GEM team (geriatrician, gerontological nurse practitioner, and social worker) and received an initial home visit, monthly clinic visits, and regular telephone contacts. The GEM team provided assessment and primary care services for an average of 6 months. 274 patients allocated to usual care received health care from their physician.

\section{Main outcome measures}

Functional ability as measured by the Sickness Impact Profile: Physical Functioning Dimension (SIP:PFD), bed disability days (BDDs), and restricted activity days (RADs); and use and cost of healthcare services (Medicare payments).

\section{Main results}

Analysis was by intention to treat. After 18 months of follow up, the GEM group had lower SIP:PFD (15.7 v 18.9 ; mean difference $3.2,95 \%$ CI 0.28 to $6.12, \mathrm{p}<0.05)$ and $\mathrm{BDD}(0.6 v 1.5$; mean difference 0.9 , CI 0.22 to 1.58 , $\mathrm{p}<0.05)$ values than the control group, but $\mathrm{RAD}$ values were similar ( $2.1 v 2.3$, mean difference 0.2 , CI -0.86 to 1.26, $\{\mathrm{p}=0.71\} \uparrow)$. More patients in the usual care group had a loss of functional ability (increase in SIP:PFD score from baseline of $\geqslant 3$ points) than in the GEM group (table). After adjusting for functional and affective status at baseline, the GEM group was less likely to lose functional ability than the usual care group (adjusted odds ratio [OR] 0.67 , CI 0.47 to 0.99 ). After adjusting for baseline use of services, the GEM group was less likely to use home care than the usual care group (adjusted OR 0.60 , CI 0.37 to 0.98 , at 12 months). Groups did not differ for nursing home use or total Medicare costs (US\$11354 v US $\$ 11786, \mathrm{p}=0.93$ ).
Randomised \{allocation concealed \} $^{*}$, blinded (outcome

\section{Conclusion}

The geriatric evaluation and management programme was more effective than usual care for preventing functional decline and minimising home care use in high risk older adults, but did not differ for nursing home use or cost of healthcare services.

*Information provided by author.

$\uparrow \mathrm{p}$ Value calculated from data in article.
Sources of funding: National Institute on Aging and the Agency for Health Care Policy and Research; the Health Care Financing Administration; the American Academy of Family Physicians Foundation.

For correspondence: Dr C Boult, Lipitz Center for Integrated Health Care,Johns Hopkins University, 624 North Broadway Street \#653, Baltimore, MD 21205, 0470 .

A modified version of this abstract appears in Evidence-Based Mental Health USA.Fax +1410955

Geriatric evaluation and management (GEM) v usual care in high risk older adults $\ddagger$

\begin{tabular}{lllll} 
Outcome at 18 months & GEM & Usual care & RRR (95\% CI) & NNT (CI) \\
Loss of functional ability & $40 \%$ & $52 \%$ & $24 \%(9$ to 37$)$ & $9(5$ to 24$)$ \\
\hline
\end{tabular}

$\ddagger$ Abbreviations defined in glossary; RRR, NNT, and Cl calculated from data in article.

\section{COMMENTARY}

The findings of this study by Boult et al are consistent with previous research that found an outpatient GEM programme to be more effective than usual care for delaying functional decline in older adults. ${ }^{1}$ In contrast to this previous study, however, Boult et al specifically targeted the intervention to a high risk group of older adults and did a cost analysis.

Despite randomisation of eligible patients, the intervention group was slightly better off than the control group in terms of health, functional ability, and previous healthcare costs. These differences may have influenced the study results. Furthermore, the authors recognised the limited scope of the financial data they were able to analyse.

Caution must be used in generalising the study results. Most patients were white $(96 \%)$ and more than half were men. Further research needs to be done to evaluate the effectiveness of this intervention with people from different races or cultures.

Although the GEM model of intervention is certainly feasible, it does involve a substantial cost (approximately US $\$ 1350$ /person). It could be argued, however, that this additional cost is warranted in view of the increased functional ability of patients. Research that explores the long term effects of the intervention would be particularly valuable, given that duration of follow up in the study by Boult et al was only 18 months.

This study highlights the valuable roles nurses can play in helping to prevent functional decline among high risk, older adults living in the community. Nurses are skilled in doing comprehensive assessments, developing and implementing care plans in collaboration with patients, and making referrals to appropriate community supports. Nurses also work collaboratively on multidisciplinary teams to provide comprehensive care to patients. These skills can be used in various practice settings (eg, family practice offices and community nursing agencies) to assist high risk older adults maintain their functional abilities and independence.

Una V Reid, RN, EdD Human Resource Development Consultant Kingston, Jamaica, West Indies Jenny Ploeg, RN, PhD Assistant Professor, School of Nursing McMaster University Hamilton, Ontario, Canada

1 Stuck AE, Aronow HU, Steiner A, et al. A trial of annual in-home comprehensive geriatric assessments for elderly people living in the community. N Engl J Med 1995;333:1184-9. 